\title{
Emerging Treatment and Prevention Strategies against COVID-19: A Brief Update
}

\author{
Ashish K. Jha ${ }^{1, \odot}$ Ravikant Kumar $^{1} \quad$ Mahesh K. Goenka ${ }^{2}$ Vishwa M. Dayal ${ }^{1}$ \\ ${ }^{1}$ Department of Gastroenterology, Indira Gandhi Institute of \\ Address for correspondence Ashish Kumar Jha, MD, DM, \\ Medical Sciences, Patna, Bihar, India \\ 2Institute of GastroSceinces, Apollo Gleneagles Hospital, Kolkata, \\ Department of Gastroenterology, Indira Gandhi Institute of Medical \\ Sciences, Sheikhpura, Bailey Road, Patna 800014, Bihar, India \\ West Bengal, India \\ (e-mail: ashishjhabn@yahoo.co.in).
}

J Digest Endosc 2020;11:69-72

\begin{abstract}
Keywords

- novel coronavirus disease 2019

- severe acute respiratory syndromecoronavirus-2

- World Health Organization

- hydroxychloroquine

- chloroquine

- remdesivir

- convalescent plasma

- vaccines

Patients with novel coronavirus disease 2019 (COVID-19) are at significantly increased risk for mortality and morbidity. Current management remains supportive care, ranging from symptomatic outpatient management to full-intensive care support, including intravenous fluids, invasive, and non-invasive oxygen supplementation. In patients with septic shock, treatment with antibiotics and vasopressors are recommended to keep mean arterial pressure (MAP) $\geq 65 \mathrm{~mm} \mathrm{Hg}$ and lactate $<2 \mathrm{mmol} / \mathrm{L}$. Because of the lack of effectiveness and possible adverse effects, routine corticosteroids should be avoided unless they are indicated for another reason (exacerbation of asthma or chronic obstructive pulmonary disease [COPD], and septic shock in whom fluids and vasopressors do not restore hemodynamic stability). There is currently no sufficient evidence of efficacy of hydroxychloroquine/chloroquine, remdesivir, and other antivirals in the treatment or prevention of COVID-19. Limited evidence shows that COVID-19 convalescent plasma can be used as a treatment of COVID-19 without the occurrence of severe adverse events. Drug regulatory agencies granted an emergency-use authorization of chloroquine/hydroxychloroquine and remdesivir to treat patients when a clinical trial is not available or participation is not feasible. Chloroquine and hydroxychloroquine are associated with QT interval prolongation and life-threatening cardiac arrhythmia in patients with pre-existing cardiovascular disease. Guidelines are issued for use of convalescent plasma in patients with serious or immediately life-threatening COVID-19. Data from several ongoing randomized controlled trials will provide further evidence regarding the safety and efficacy of these drugs for the treatment of COVID-19.
\end{abstract}

\section{Introduction}

The current novel coronavirus disease 2019 (COVID-19) pandemic, caused by a novel severe acute respiratory syndrome-coronavirus-2 (SARS-CoV-2), is a rapidly spreading viral disease. Patients with COVID-19 are at significantly increased risk for mortality and morbidity. Since the first report of SARS-CoV-2 infection, it has spread to more than 210 countries around the world (2,726,776 active cases and 191,087 death [till April 24, 2020]). To date, India has recorded 23,502 active cases and 722 death (till April 24, 2020). There is currently no specific treatment of COVID-19. Current management remains supportive care, ranging from symptomatic outpatient management to full intensive care support, including intravenous fluids, invasive and noninvasive oxygen supplementation, and antibiotics. ${ }^{1}$ The objective of this paper is to briefly review the literature and update the concept of prevention and treatment of COVID-19. We have highlighted here the potential therapeutic role of remdesivir, chloroquine/ hydroxychloroquine (HCQ), lopinavir/ritonavir, and convalescent plasma in patients with SARS-CoV-2 infection.
DOI https://doi.org/

10.1055/s-0040-1712547

ISSN 0976-5042.
License terms

(요 (1) $\Theta \circledast$ 


\section{Standard of Care: World Health Organization Recommendations}

In the absence of a proven therapy for SARS-CoV 2, the cornerstone of therapy for patients with COVID-19 remains supportive care. The standard of care management of COVID-19 are as follows:

- Early recognition of patients with SARS infection associated with COVID-19 and immediate implementation of infection prevention and control measures.

- Collection of specimens including blood cultures, specimens from the upper respiratory tract, and the lower respiratory tract (when needed).

- Mild COVID-19 should be managed with symptomatic treatment and monitoring. Management of severe COVID19 includes intravenous fluids, oxygen therapy (saturation target $>94 \%$ ), and monitoring. Associated coinfections should be treated with antibiotics.

- Advanced oxygen/ventilatory support is warranted in patients of critical COVID-19 with acute respiratory distress syndrome (ARDS)/severe hypoxemic respiratory failure. Mechanical ventilation using lower tidal volumes (4-8 $\mathrm{mL} / \mathrm{kg}$ predicted body weight [PBW]) and lower inspiratory pressures (plateau pressure $<30 \mathrm{~cm} \mathrm{H}_{2} \mathrm{O}$ ) is preferred. In adult patients with severe ARDS, prone ventilation for 12 to 16 hours per day is recommended. ARDS patients without tissue hypoperfusion are treated with conservative fluid management strategy.

- In patients with septic shock, treatment with antibiotics and vasopressors are recommended to keep mean arterial pressure (MAP) $\geq 65 \mathrm{~mm} \mathrm{Hg}$ and lactate $<2 \mathrm{mmol} / \mathrm{L}$.

- The rationale to use corticosteroid in severe COVID-19 is to suppress the inflammatory response which may lead to acute lung injury and ARDS. In a retrospective study ( $n=201)$, treatment with methylprednisolone was associated with a decreased risk of death (46\% with steroids vs. $62 \%$ without). ${ }^{2}$ However, the authors noted that confounding bias may exist in this observational study. Because of the lack of effectiveness and possible adverse effects, routine corticosteroids should be avoided unless they are indicated for another reason (exacerbation of asthma or COPD and septic shock in whom fluids and vasopressors do not restore hemodynamic stability). ${ }^{1,3}$

\section{Specific Treatments with Potential Clinical Benefit}

\section{Remdesivir}

Remdesivir is a nucleotide analogue prodrug that inhibits viral RNA polymerases. It has broad-spectrum activity against RNA viruses such as coronaviridae (e.g., SARS-CoV and Middle East respiratory syndrome coronavirus [MERS- CoV]) and filoviruses (e.g., Ebola). It has shown prophylactic and therapeutic efficacy in nonclinical models of these coronaviruses; however, there are currently only very limited data on the use of remdesivir in patients with COVID-19. In a recent multicentric study, patients with confirmed SARS-CoV-2 infection $(n=53)$ who had an oxygen saturation of $94 \%$ or less with or without receiving oxygen support were enrolled. Besides supportive care, patients received remdesivir, consisting of $200 \mathrm{mg}$ administered intravenously on day 1 , followed by $100 \mathrm{mg}$ daily up to 10 days. During a median follow-up of 18 days, $68 \%$ had an improvement in oxygen-support class, including $57 \%$ patients receiving mechanical ventilation. However, interpretation of the result of this study is limited by the lack of a randomized control group, small sample size, exclusion of serious cases (creatinine clearance $<30 \mathrm{~mL} / \mathrm{min}$ and >five-time elevation of serum aminotransferase), variable duration of remdesivir administration, noncollection of viral load data, adverse effects, and short-term follow-up. ${ }^{4} \mathrm{~A}$ total of $60 \%$ patients reported adverse events during follow-up. The result of a study (SIMPLE trial; unpublished) demonstrated that patients receiving a 10-day course of remdesivir achieved similar clinical improvement compared with those taking a 5-day treatment course on Day 14. The European Medicines Agency (EMA) has published recommendations on compassionate use of remdesivir for COVID-19.5 Data from several ongoing randomized controlled trials will provide further evidence regarding the safety and efficacy of remdesivir for COVID-19.

\section{Chloroquine and Hydroxychloroquine}

The antimalarial and anti-inflammatory agents, chloroquine and HCQ appear to block viral entry into cells by inhibiting glycosylation of host receptors, proteolytic processing, and endosomal acidification. These agents also have immunomodulatory effects through attenuation of cytokine production and inhibition of autophagy and lysosomal activity in host cells. ${ }^{6,7}$ Both drugs have in vitro activity against SARSCoV-2, with HCQ having relatively higher potency.

Early results obtained from more than 100 patients enrolled in studies conducted in the China showed the superiority of chloroquine compared with the controls in terms of reduction of exacerbation of pneumonia, duration of symptoms, and delay of viral clearance, all in the absence of severe side effects. ${ }^{8}$ A recent open-label study of 36 patients reported improved virologic clearance with HCQ $(n=36)$ compared with the controls $(n=20)$. Addition of azithromycin to HCQ in six patients resulted in superior viral clearance (100\%) compared with HCQ monotherapy (57\%). ${ }^{9}$ An uncontrolled observational study in a cohort of COVID-19 inpatients $(n=80)$ treated with a combination of HCQ and azithromycin showed a rapid fall of nasopharyngeal viral load (93\% at day 8$).^{2}$ In a randomized parallel-group trial, COVID-19 patients $(n=31)$ were assigned to receive an additional 5-day HCQ (400 mg/d) treatment. Clinical recovery time was significantly shortened in the HCQ treatment group. Improvement in pneumonia was more in the HCQ treatment group (80.6\%) compared with the controls (54.8\%). However, the patients had mild disease. ${ }^{10}$

In a prospective study ( $n=30)$, patients were randomized to HCQ (400 mg/day for 5 days) plus standard of care and standard care alone groups. Virologic clearance was similar in both the groups. ${ }^{11} \mathrm{~A}$ study of 11 patients with COVID-19 reported persistence of SARS-CoV-2 in the nasopharyngeal swab in 8 of 10 patients receiving HCQ. In a randomized, double-blinded, phase-IIb clinical trial (CloroCOVID-19 Study), 
preliminary findings suggest that the higher chloroquine dosage is associated with higher rate of QTc prolongation and mortality. Studies of chloroquine prophylaxis in health care workers (NCT04303507) and HCQ for postexposure prophylaxis after high-risk exposures (NCT04308668) are planned or enrolling. ${ }^{12}$

Some studies show no benefit at all, while others show a benefit of taking $\mathrm{HCQ} /$ chloroquine. Some of the aforementioned clinical studies showed $\mathrm{HCQ} /$ chloroquine as promising agents for the treatment of mild-to-severe COVID-19. However, these trials have been criticized for their limitations, including small sample size, nonuniform study design, absence of control arm, and enrolment of less severe cases. ${ }^{2,9,10,13}$ Results from other clinical studies could not replicate these positive findings. ${ }^{11,12,14}$ Therefore, there is currently no strong evidence of efficacy of HCQ or chloroquine in the treatment or prevention of COVID-19. Chinese and Italian guidelines recommend early use of HCQ or chloroquine for the treatment of COVID-19 on case-to-case basis; however, this is based on weak evidence. The Food and Drug Administration (FDA) and EMA has granted an emergency-use authorization for chloroquine and HCQ to treat patients when a clinical trial is not available or participation is not feasible. ${ }^{5,15}$ Indian Council of Medical Research (ICMR) recommends the use of HCQ for prophylaxis of SARS CoV-2 infection in high-risk cases. QT-prolongation and life-threatening cardiac arrhythmia is major concern in patients receiving HCQ with or without azithromycin, especially in patients with preexisting cardiovascular disease.

\section{Lopinavir/Ritonavir}

Lopinavir/ritonavir demonstrated in vitro activity against other corona viruses via inhibition of 3-chymotrypsin-like protease. Results of a few small case series showed clinical benefit of using with lopinavir/ritonavir in patients with COVID-19. However, an open-label randomized trial at a single hospital concluded that lopinavir/ritonavir recipients and those receiving standard care did not differ significantly in time to clinical improvement (median, 16 days), duration of intensive care unit stay, and duration of mechanical ventilation/oxygen supplementation. ${ }^{16}$ At present, there is insufficient evidence to recommend the use of lopinavir/ritonavir for treatment of COVID-19.

\section{Convalescent Plasma}

Convalescent plasma from patients who have recovered from viral infections has been used as a treatment in previous virus outbreaks including SARS and Ebola virus. Convalescent plasma or immunoglobulins were used in patients with SARS whose condition continued to deteriorate despite treatment with intravenous corticosteroids. A systematic review and exploratory meta-analysis from 32 studies of SARS coronavirus infection and severe influenza showed a statistically significant reduction in the pooled odds of mortality following treatment with convalescent plasma compared with placebo (odds ratio $=0.25$; 95\% confidence interval $[\mathrm{CI}]: 0.14-0.45 ; \mathrm{I}[2]=0 \%$ ). Study showed consistent evidence for a reduction in mortality, especially when convalescent plasma is administered early after symptom onset. No serious adverse events were seen. However, studies were commonly of low or very low quality, lacked control groups, and at moderate or high risk of bias. ${ }^{17}$ Luke et al identified eight studies involving 1,703 patients with Spanish influenza pneumonia treated with influenza-convalescent human blood products infusion, which showed a pooled absolute reduction of $21 \%$ $(p<0.001)$ in the overall crude case-fatality rate. Mortality rate was lower in patients who received early treatment (after $<4$ days of complications) compared with late treatment group (after $>4$ days of complications; 26 vs. $50 \%$ [pooled risk difference $=41 \%(\mathrm{CI}: 29-54 \%)])^{18}$

Convalescent plasma has also been tried in treatment of COVID-19. A few small preliminary case series showed clinical improvement after treatment with convalescent plasma; however, these studies had many limitations. ${ }^{19,20}$ Limited evidence shows that convalescent plasma can be used as a treatment of COVID-19 without the occurrence of severe adverse events. ${ }^{19}$ Convalescent plasma that contains antibodies to SARS-CoV-2 might suppress viremia. The patient usually develops a primary immune response by days 10 to 14 , which is followed by virus clearance. Therefore, logically, it should be more effective to transfuse the convalescent plasma at the early course of disease. Studies showed lower mortality rate in whom convalescent plasma is administered early after symptom onset. ${ }^{17,18}$ The FDA has issued guidance for use of COVID-19 convalescent plasma in patients with serious COVID-19. About 3 to 4 weeks after a person recovers, blood can be drawn to look for the antibodies. The approach involves giving COVID-19 patients an infusion of antibody-rich plasma (single dose of $200 \mathrm{~mL}$ ) from people who have recovered from an infection with SARS-CoV-2.

\section{Future Prevention/Treatment}

The rapidly expanding knowledge regarding SARS-CoV-2 virology provides a significant number of potential drug targets. Potential mechanisms of action of antiviral agents are (1) virus-based therapy, like monoclonal antibodies or antiviral peptides, targeting the various steps of viral machinery-like spike glycoprotein, viral enzyme inhibitors, and viral nucleic acid synthesis inhibitors, and (2) host-based therapies to potentiate the interferon response, affect host signalling pathways, or host factors utilized by SARS-CoV-2 for replication. ${ }^{21}$ The World health Organization (WHO) has started an international multicentric clinical trial ("Solidarity"). This trial comprises of four treatment groups (remdesivir, chloroquine/hydroxychloroquine, lopinavir with ritonavir, and lopinavir with ritonavir plus interferon $\beta-1 a$ ) against standard of care, to assess their relative effectiveness against COVID-19. ${ }^{1}$ Several therapies are currently being investigated globally, including nitazoxanide, stem cell therapy, intravenous immunoglobulin, interleukin-6 receptor antagonist (e.g., tocilizumab, sarilumab, and siltuximab), Bacille Calmette-Guerin (BCG) vaccine, angiotensin-II receptor antagonists, and other antivirals (e.g., oseltamivir, danoprevir, darunavir, ganciclovir, favipiravir, baloxavir 
marboxil, umifenovir, sofosbuvir, ribavirin, and interferon alfa/ $\beta)$.

\section{Vaccine: A Ray of Hope for COVID-19}

The most effective long-term strategy for prevention of future outbreaks of this virus would be the development of a vaccine providing protective active immunity. As of April 8, 2020, the global COVID-19 vaccine research and development landscape included 115 vaccine candidates. Out of 78 confirmed active projects, 73 are currently at preclinical stages. ${ }^{22} \mathrm{~A}$ few vaccine candidates have moved into clinical trial phase, including mRNA-1273 (Moderna), Ad5-nCoV (CanSino Biologicals), INO-4800 (Inovio), LV-SMENP-DC, and pathogen-specific aAPC (Shenzhen Geno-Immune Medical Institute). Unfortunately, a vaccine is unlikely to be ready for use before the end of 2020 or 2021 at the earliest.

\section{Conclusion}

WHO and Centers for Disease Control and Prevention guidance emphasizes the role of supportive care based on severity of illness, ranging from symptomatic treatment for mild disease to evidence-based ventilator management for ARDS, and early recognition and treatment of bacterial infections and sepsis in critically ill patients. Use of routine systematic corticosteroids should be avoided for the treatment of COVID-19. There is currently no strong evidence of efficacy of $\mathrm{HCQ} /$ chloroquine and remdesivir or other antivirals in the treatment or prevention of COVID-19. The FDA and EMA has granted an emergency-use authorization for chloroquine/ HCQ to treat patients when a clinical trial is not available or participation is not feasible. The ICMR recommends the use of HCQ for prophylaxis of SARS-CoV-2 infection in high-risk cases. The EMA recommends use of remdesivir for COVID-19 on a compassionate basis. Guidelines are issued for use of convalescent plasma in patients with serious or immediately life-threatening COVID-19. Data from several ongoing randomized controlled trials will provide further evidence regarding the safety and efficacy of these drugs for COVID-19.

\section{Conflict of Interest}

None declared.

\section{References}

1 World Health Organization. Country \& Technical Guidance Coronavirus disease (COVID-19). Available at:https://www. who.int/emergencies/diseases/novel-coronavirus-2019/technical-guidance Accessed April 19, 2020

2 Gautret P, Lagier JC, Parola P, et al. Clinical and microbiological effect of a combination of hydroxychloroquine and azithromycin in 80 COVID-19 patients with at least a six-day follow up: a pilot observational study. Travel Med Infect Dis 2020; (e-pub ahead of print). doi 10.1016/j.tmaid.2020.101663

3 Wu C, Chen X, Cai Y, et al. Risk factors associated with acute respiratory distress syndrome and death in patients with corona virus disease 2019 pneumonia in Wuhan, China. JAMA Intern Med 2020; (e-pub ahead of print). doi 10.1001/ jamainternmed.2020.0994
4 Grein J, Ohmagari N, Shin D, et al. Compassionate use of remdesivir for patients with severe COVID-19. N Engl J Med 2020; (e-pub ahead of print). doi 10.1056/NEJMoa2007016

5 European Medicines AgencySummary on compassionate use. Available at: https://www.ema.europa.eu/en/documents/other/summary-compassionate-use-remdesivir-gilead_en.pdf. Accessed April 19, 2020)

6 Zhou D, Dai SM, Tong Q. COVID-19: a recommendation to examine the effect of hydroxychloroquine in preventing infection and progression. J Antimicrob Chemother 2020; (e-pub ahead of print). doi 10.1093/jac/dkaa114.10

7 Devaux CA, Rolain JM, Colson P, Raoult D. New insights on the antiviral effects of chloroquine against coronavirus: what to expect for COVID-19? Int J Antimicrob Agents 2020; (e-pub ahead of print). doi 10.1016/j.ijantimicag.2020.105938

8 Gao J, Tian Z, Yang X. Breakthrough: Chloroquine phosphate has shown apparent efficacy in treatment of COVID-19 associated pneumonia in clinical studies. Biosci Trends 2020;14(1):72-73

9 Gautret P, Lagier JC, Parola P, et al. Hydroxychloroquine and azithromycin as a treatment of COVID-19: results of an open-label non-randomized clinical trial. Int J Antimicrob Agents 2020; (e-pub ahead of print). doi 10.1016/j.ijantimicag.2020.105949

10 Chen Z, Hu J, Zhang Z, et al. Efficacy of hydroxychloroquine in patients with COVID-19: results of a randomized clinical trial. Med Rxiv 2020; doi 10.1101/2020.03.22.20040758

11 Chen J, Liu D, Liu L, et al. A pilot study of hydroxychloroquine in treatment of patients with common coronavirus disease-19 (COVID-19) J Zhejiang Univ (Med Sci) 2020;49(1doi 10.3785/j. issn.1008-9292.2020.03.03

12 Kalil AC. Treating COVID-19-off-label drug use, compassionate use, and randomized clinical trials during pandemics. JAMA 2020;(e-pub ahead of print). doi 10.1001/jama.2020.4742

13 Taccone FS, Gorham J, Vincent JL. Hydroxychloroquine in the management of critically ill patients with COVID-19: the need for an evidence base. Lancet Respir Med 2020; (e-pub ahead of print). doi 10.1016/S2213-2600(20)30172-7

14 Molina JM, Delaugerre C, Le Goff J, et al. No evidence of rapid antiviral clearance or clinical benefit with the combination of hydroxychloroquine and azithromycin in patients with severe COVID-19 infection. Med Mal Infect 2020; (e-pub ahead of print). doi 10.1016/j.medmal.2020.03.006

15 U.S. Food and Drug AdministrationRequest for Emergency Use Authorization For Use of Chloroquine Phosphate or Hydroxychloroquine Sulfate Supplied From the Strategic National Stockpile for Treatment of 2019 Coronavirus Disease. https:// www.fda.gov/media/136534/download Accessed April 19, 2020

16 Cao B, Wang Y, Wen D, et al. A trial of lopinavir-ritonavir in adults hospitalized with severe COVID-19. N Engl J Med 2020; (e-pub ahead of print). doi 10.1056/NEJMoa2001282

17 Mair-Jenkins J, Saavedra-Campos M, BaillieJK, et al; Convalescent Plasma Study Group. The effectiveness of convalescent plasma and hyperimmune immunoglobulin for the treatment of severe acute respiratory infections of viral etiology: a systematic review and exploratory meta-analysis. J Infect Dis 2015;211(1):80-90

18 LukeTC, Kilbane EM,JacksonJL, Hoffman SL. Meta-analysis: convalescent blood products for Spanish influenza pneumonia: a future H5N1 treatment? Ann Intern Med 2006;145(8):599-609

19 Chen L, Xiong J, Bao L, Shi Y. Convalescent plasma as a potential therapy for COVID-19. Lancet Infect Dis 2020;20(4):398-400

20 Shen C, Wang Z, Zhao F, et al. Treatment of 5 critically ill patients with COVID-19 with convalescent plasma. JAMA 2020;(e-pub ahead of print) doi:10.1001/jama.2020.4783

21 Zumla A, Chan JF, Azhar EI, Hui DS, Yuen KY. Coronaviruses drug discovery and therapeutic options. Nat Rev Drug Discov 2016;15(5):327-347

22 Thanh Le T, Andreadakis Z, Kumar A, et al. The COVID-19 vaccine development landscape. Nat Rev Drug Discov 2020; (e-pub ahead of print). doi 10.1038/d41573-020-00073-5 